\title{
Intégration de la géométrie d'outil dans la prédiction des efforts de coupe en fraisage de matériaux durs
}

\author{
Stéphanie Bissey ${ }^{1, a}$, Gérard Poulachon ${ }^{1, a}$ Et François Lapujoulade $^{2}$ \\ 1 LaBoMaP, ENSAM, Rue Porte de Paris, 71250 Cluny, France \\ 2 LMSP, ENSAM, 151 Bd. de l'hôpital, 75013 Paris, France
}

Reçu le 14 février 2005, accepté le 13 avril 2005

\begin{abstract}
Résumé - La connaissance des efforts de coupe grâce à un modèle prédictif est très intéressante pour le choix de la puissance d'une machine-outil, des outils coupants, l'optimisation des conditions de coupe, le contrôle de l'apparition de vibrations, ou la surveillance d'outil « on-line ». Cela permettrait de diminuer le nombre d'essais, et de déterminer la meilleure géométrie d'outil en fonction d'efforts limites fixés. L'objectif de cette étude est la prédiction de l'influence des angles de coupe et d'hélice sur les variations d'efforts de coupe, et de les intégrer dans une relation de coupe. Des essais ont été menés dans l'acier à moules X38 CrMoV 5 (AISI H11), avec des fraises carbure monobloc d'angles de coupe différents et à denture droite. Une configuration spécifique a été utilisée pour créer l'obliquité. Les efforts de coupe ont été mesurés à différentes avances. Les résultats ont permis de confirmer le modèle d'efforts de coupe et de l'appliquer sur des essais de fraisage proprement dits.
\end{abstract}

Mots clés : Efforts de coupe / fraisage / angle de coupe / angle d'hélice / matériau dur

\begin{abstract}
Integration of tool geometry in prediction of cutting forces during milling of hard materials. The knowledge of cutting forces thanks to a predictive model is very interesting for the choice of a machine tool power, the cutting tools, the optimization of cutting conditions or the control of the occurence of vibrations. It could also be helpful in on-line wear monitoring during milling. This could allow to decrease the number of tests, and to determine the best tool geometry, in relation with a limit level of forces. The objective of the study is the prediction of effects of rake and helix angles on cutting forces variations, and to integrate it in a cutting relation. Tests were made on X $38 \mathrm{CrMoV} 5$ (AISI H11) die and mold steel, with solid carbide milling cutters of various rake angles and straight flutes. A specific configuration has been used to create obliquity. Cutting forces were measured for various feed rates. The results have improved the cutting force model, and allowed to apply it to "real" milling configurations.
\end{abstract}

Key words: Cutting forces / milling / rake angle / helix angle / hard material

\section{Introduction}

Dans le contexte actuel de la recherche de hauts niveaux de qualité des produits fabriqués, la possibilité d'obtenir des prédictions réalistes des valeurs d'efforts de coupe devient très importante. En effet, connaître le niveau des efforts de coupe pendant une opération d'usinage permettrait de réduire le nombre d'essais longs et coûteux dont le but est la détermination des meilleures conditions de coupe et de la meilleure géométrie d'outil pour une opération d'usinage donnée. Plusieurs travaux

\footnotetext{
a Auteurs pour correspondance:

stephanie.bissey@cea.fr

gerard.poulachon@cluny.ensam.fr
}

de recherche sur la « mécanique » du phénomène de coupe peuvent être cités. Une première approche est de relier la coupe orthogonale et la coupe oblique [1-3]. Ces travaux ont été repris plus récemment, en considérant les données de la coupe orthogonale comme une base de données pour la modélisation des efforts en coupe oblique $[4,5]$. Armarego et Deshpande [6-8] ont présenté une comparaison entre plusieurs modèles de prédiction d'efforts en fraisage : le « cas idéal », le modèle avec « excentricité » et celui avec « flexion » qui nécessite de connaître certaines grandeurs telles que la contrainte de cisaillement, le rapport de compression ou l'angle de frottement pour le matériau usiné considéré. Le modèle utilisé par Altintas [4] inclut les modifications dans la répartition de 


\section{Nomenclature}

\begin{tabular}{|c|c|}
\hline$a_{\mathrm{p}}, a_{\mathrm{e}}$ & profondeur de passe axiale, radiale \\
\hline$b, h, l$ & largeur, épaisseur et longueur de coupe \\
\hline$\Delta z$ & épaisseur d’un élément de l'outil \\
\hline$f_{z}$ & avance par dent \\
\hline$F_{i j}$ & effort de coupe local sur la $i^{\text {ème }}$ dent, le $j$ ème élément \\
\hline $\begin{array}{l}F_{k} \\
(\vec{g}, \vec{n}, \vec{a})\end{array}$ & $\begin{array}{l}\text { effort de coupe dans la direction } k ; k=x, y, z, r, t, a, g, n \ldots \\
\text { repère lié à la face de coupe }\end{array}$ \\
\hline$\gamma$ & angle de coupe \\
\hline$\lambda_{\mathrm{e}}, \lambda_{\mathrm{n}}$ & angle d'écoulement du copeau, angle entre $P Q$ et $P R$ (Fig. 1) \\
\hline$\lambda_{\mathrm{s}}$ & angle d'hélice \\
\hline$M$ & nombre d'éléments \\
\hline$N$ & vitesse de rotation de la broche \\
\hline$P_{i j}$ & centre du segment $S_{i j}$ \\
\hline$S_{i j}$ & segment sur la $i^{\text {ème }}$ dent, le $j$ ème élément \\
\hline$V_{\mathrm{c}}$ & vitesse de coupe \\
\hline
\end{tabular}

pression sur la face de coupe dues aux vibrations, ainsi que la géométrie d'arête, et l'intensité des efforts de coupe et leur direction sont déterminées grâce à la transformation orthogonale-oblique proposée par Armarego et al. [9]. D'autres auteurs ont également examiné le problème de l'estimation des efforts en fraisage. Plusieurs modèles ont été développés en accentuant plus ou moins des points particuliers : Jayaram et al. [10] ont donné une estimation des efforts spécifiques de coupe pour un modèle d'efforts en fraisage en bout, Junz Wang et al. [11] ont proposé un modèle d'efforts analytique prenant en compte les effets du cisaillement et d'arête en fraisage mixte. Un modèle pour les fraises hélicoïdales, basé sur le principe de la segmentation et utilisant le modèle d'Oxley [12] a été présenté par Li et al. [13]. Les fraises hélicoïdales ont également intéressé Altintas et al. [14], qui ont proposé un modèle généralisé pour ces fraises, prenant en compte la cinématique de la coupe avec ou sans vibrations. Lapujoulade [15] a étudié plus particulièrement la finition de voiles minces en termes d'efforts de coupe et de lobes de stabilité. Ko et al. [16] ont pris en considération l'effet de la taille de l'arête et ont essayé de déterminer des coefficients indépendants des conditions de coupe.

L'objectif de cette étude est la prédiction des effets des angles de coupe et d'hélice sur les efforts de coupe, afin de les intégrer dans une loi de coupe. Ainsi, le nombre d'essais avant une opération de fraisage pourrait être limité et le choix d'angles de coupe et d'hélice optimaux pour une opération donnée pourrait être possible.

\section{Formulation du modèle}

Le modèle théorique présenté dans cet article est basé sur le principe bien connu de la segmentation $[13,15,16]$. L'outil est divisé en $M$ éléments d'épaisseur constante $\Delta z$, perpendiculairement à son axe, et les composantes de l'effort de coupe s'appliquant sur l'arête à un instant donné sont obtenues par sommation des composantes des efforts s'appliquant sur chaque élément. Finalement, une sommation sur toutes les arêtes engagées dans la matière

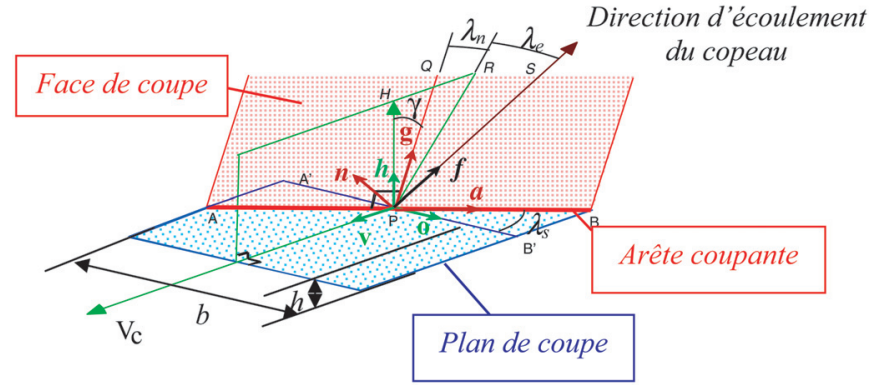

Fig. 1. Modélisation de la coupe oblique.

permet d'obtenir l'effort global s'appliquant sur l'outil à un instant donné. Ainsi, les arêtes coupantes sont divisées en segments de longueur constante. $P_{i j}$ représente le milieu de $S_{i j}$, segment de la $i$ ème $\operatorname{dent}$ et du $j$ ème élément. L'épaisseur de coupe axiale et l'engagement radial seront notés respectivement $a_{\mathrm{p}}$ et $a_{\mathrm{e}}$. Plusieurs systèmes de coordonnées sont utilisés afin de représenter la coupe oblique, d'exprimer les efforts sur chaque segment dans un repère local puis d'effectuer les sommations dans un repère global lié à l'outil.

\subsection{Modélisation de la coupe oblique}

La figure 1 représente une modélisation de la coupe oblique avec les différents repères utilisés.

Le système de coordonnées $(\vec{g}, \vec{n}, \vec{a})$ est lié à la face de coupe et constitue un repère intermédiaire pour le calcul et la détermination de la loi de coupe. L'effort de coupe est contenu dans le plan dont la trace sur la face de coupe est $P S$, c'est-à-dire la direction d'écoulement du copeau, donc : $\vec{F}=F_{\mathrm{n}} \cdot \vec{n}+F_{\mathrm{f}} \cdot \vec{f}$ avec $\vec{f}$ suivant $P S$. L'effort de coupe est caractérisé par le triplet $\left\{F_{\mathrm{n}}, F_{\mathrm{f}}, \lambda_{\mathrm{e}}\right\}$, avec $\lambda_{\mathrm{e}}$ angle d'écoulement du copeau. Une loi de coupe doit exprimer l'évolution de $F_{\mathrm{n}}, F_{\mathrm{f}}$ et $\lambda_{\mathrm{e}}$ en fonction de l'épaisseur de coupe $h$, l'angle de coupe $\gamma$, l'angle d'hélice $\lambda_{\mathrm{s}}$, la vitesse de coupe $V_{\mathrm{c}}$ et la largeur de coupe $b$ ou la longueur d'arête en prise $l=A B$. 


\subsection{Loi de coupe oblique}

\section{Paramètres}

Les différents paramètres à inclure dans le modèle pour tenir compte des lois physiques du phénomène de coupe et de la géométrie d'outil doivent être examinés. Les principaux paramètres sont l'épaisseur de coupe $(h)$, l'angle d'hélice $\left(\lambda_{\mathrm{s}}\right)$, la largeur de coupe $(b)$, la longueur d'arête en prise $(l)$, la vitesse de coupe $\left(V_{\mathrm{c}}\right)$, l'angle de coupe $(\gamma)$.

- L'épaisseur de coupe $h$ et la largeur de coupe $b$ forment la section coupée et, par conséquent, ne peuvent être ignorées.

- La vitesse de coupe a peu d'influence [6-8] et peut être négligée si elle varie peu. Toutefois, elle doit être prise en compte si la géométrie d'outil entraîne de faibles vitesses de coupe dans certaines zones (près de l'axe de rotation par exemple) car le phénomène de formation du copeau peut changer de manière significative. Dans notre étude, tous les essais ont été menés avec une vitesse de coupe comprise dans le domaine de fonctionnement du couple outil-matière au sens de la norme du C.O.M. [17].

- L'angle d'obliquité $\lambda_{\mathrm{s}}$ doit évidemment apparaître dans la loi de coupe oblique (fraises hélicoïdales).

- L'angle de coupe $\gamma$ est intéressant à étudier pour les cas où il varie le long de l'arête ou pour comparer des outils entre eux. De plus, la décomposition des efforts dans le repère lié à la face de coupe donne lieu à des composantes d'efforts plus physiquement interprétables.

- La longueur d'arête en prise pourrait être utilisée à la place de la largeur de coupe mais la notion de section coupée disparaît, ou en complément à l'épaisseur de coupe pour caractériser l'action de l'arête elle-même $\left(r_{\beta}\right)$.

\section{Composantes de l'effort de coupe}

L'effort de coupe peut être exprimé dans n'importe quel repère. Toutefois, la complexité de la loi de coupe dépend du choix du repère dans lequel elle est exprimée : il est plus simple de séparer les influences des différents paramètres si le repère est judicieusement choisi. Le référentiel lié à la face de coupe $(\vec{g}, \vec{n}, \vec{a})$ permet de bien séparer la composante normale due à la pression exercée par le copeau sur la face de coupe et la composante tangentielle liée au frottement lors du déplacement du copeau sur la face de coupe. La relation de coupe sera donc exprimée dans ce repère lié à la face de coupe de l'outil grâce à :

- la composante normale à la face de coupe $F_{\mathrm{n}}$;

- la composante tangentielle à la face de coupe $F_{\mathrm{f}}$;

- la direction d'écoulement du copeau caractérisée par l'angle $\lambda_{\mathrm{e}}$.

\section{Loi de coupe oblique}

Une loi de coupe oblique doit prendre en compte le cas particulier de la coupe orthogonale. Il est connu que l'influence de l'épaisseur de coupe sur l'effort de coupe est non-linéaire pour ses faibles valeurs et est quasilinéaire pour ses fortes valeurs. Une des meilleures formes représentative est :

$$
F_{\mathrm{n}}=\left(K_{\mathrm{n}_{0}}+K_{\mathrm{n}_{1}} \cdot\left(h-h_{0}\right)\right) \cdot b \quad \text { pour } \quad h \geq h_{0}
$$

- une évolution linéaire ou parabolique pour $0 \leq h \leq h_{0}$, où $h_{0}$ est la limite entre les deux zones d'évolution.

L'angle de coupe intervient dans la loi de coupe par l'intermédiaire d'un terme correctif : $\left(1+K_{n \gamma} \cdot \gamma\right)$. La composante $F_{\mathrm{f}}$ est exprimée à partir de la partie linéaire de l'expression de la composante normale et du coefficient de frottement $C_{\mathrm{f}}$.

$$
\begin{aligned}
F_{\mathrm{f}} & =C_{\mathrm{f}} \cdot\left(F_{\mathrm{n}}-F_{\mathrm{n} 0}\right)+F_{\mathrm{f} 0} \\
& =K_{\mathrm{f}_{0}} \cdot b+C f \cdot K_{\mathrm{n} 1} \cdot\left(h-h_{0}\right) \cdot b \cdot\left(1+K_{\mathrm{n} \gamma} \cdot \gamma\right)
\end{aligned}
$$

avec $F_{\mathrm{n} 0}=K_{\mathrm{n} 0} . b$ et $F_{\mathrm{f} 0}=K_{\mathrm{f} 0} \cdot b$.

La mise en place du modèle oblige à utiliser une méthode d'identification car le calcul direct est impossible si des équations linéaires ne peuvent être considérées. Ceci explique pourquoi une méthode directe est utilisée afin d'isoler un segment d'arête et retrouver les mêmes conditions sur toute sa longueur. Pour déterminer les coefficients d'efforts de coupe, et ainsi l'expression de la loi de coupe, une série d'essais a été menée dans la configuration présentée dans la section suivante.

\section{Procédure expérimentale des essais préliminaires}

L'objectif est de déterminer une loi de coupe utilisable avec n'importe quelle fraise de la même « famille » que les outils testés (même matériau, même préparation d'arête ... ) même si, par exemple, l'angle d'hélice varie le long de l'arête. Le but de ces essais est de se placer dans des conditions plus simples d'exploitation que pour des essais de fraisage, afin d'étudier séparément les influences des angles de coupe et d'hélice puis de les intégrer dans la loi de coupe. De plus, pour des raisons économiques, le nombre de fraises à fabriquer a été minimisé. Le matériau usiné choisi est un acier à moules et matrices X 38 CrMoV 5 (AISI H11) durci à 43 HRc. Les outils testés sont des fraises carbure monobloc à denture droite, 2 dents, de diamètre $20 \mathrm{~mm}$, sans préparation d'arête, chacune avec un angle de coupe différent. Les essais ont été réalisés sur une fraiseuse 5 axes GAMBIN $120 \mathrm{CR}$ et sur un centre d'usinage DMG (DMC $65 \mathrm{~V})$.

Les conditions de coupe (Tab. 1) ont été choisies après avoir effectué la première partie de la méthodologie du COM [17], qui permet de définir les meilleures conditions de coupe en termes de coefficients spécifiques de coupe et de stabilité (la zone obtenue pour la vitesse de coupe est : $\left.90 \leq V_{\mathrm{c}} \leq 150 \mathrm{~m} \cdot \mathrm{min}^{-1}\right)$.

La configuration d'essais est comparable à du tournage (Fig. 2). L'outil est fixé sur la platine dynamométrique et la pièce est entraînée en rotation grâce au mouvement de broche. Étant donné que les fraises 
Tableau 1. Conditions expérimentales pour les deux séries d'essais.

\begin{tabular}{ccc}
\hline Conditions & $\begin{array}{c}\text { Influence de l'angle } \\
\text { de coupe }\end{array}$ & $\begin{array}{c}\text { Influence de l'angle } \\
\text { d'hélice }\end{array}$ \\
\hline expérimentales & disque & tube \\
Forme de la pièce & 140 & 70 \\
Diamètre extérieur $(\mathrm{mm})$ & 110 & 127 \\
$V_{\mathrm{c}}\left(\mathrm{m}_{\mathrm{min}}{ }^{-1}\right)$ & 0,01 to 0,2 & 0,1 \\
$f\left(\mathrm{~mm}_{\mathrm{dent}}{ }^{-1}\right)$ & 0 & $0,10,20,30,40,50,60$ \\
$\lambda_{\mathrm{s}}\left(^{\circ}\right)$ & $-4,0,4,8,12,16,20$ & $8,12,16$ \\
$\gamma\left({ }^{\circ}\right)$ &
\end{tabular}
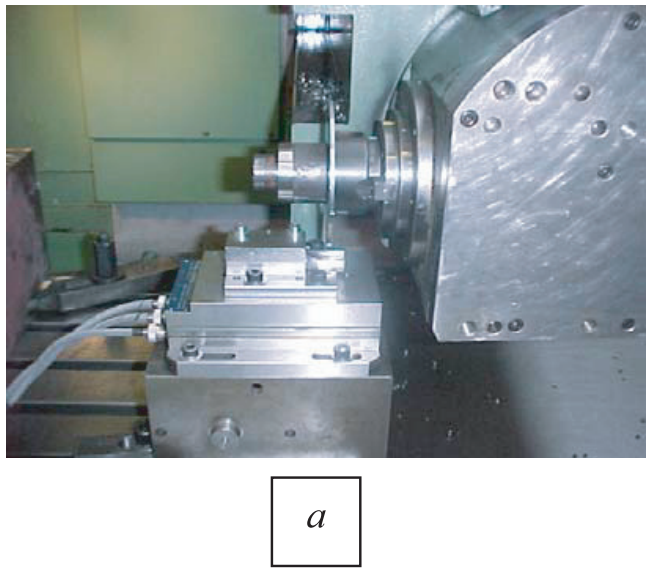

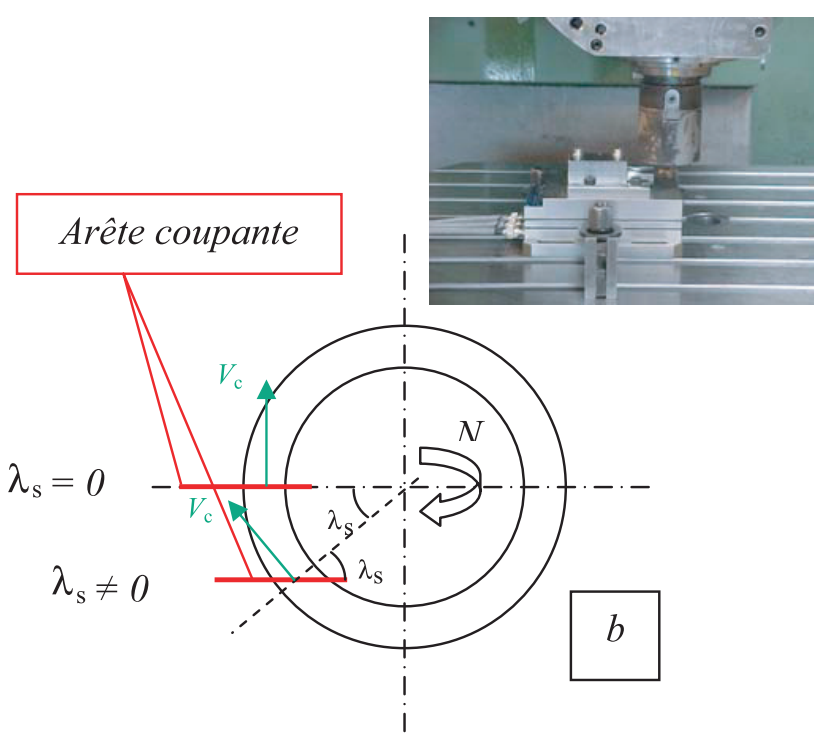

Fig. 2. Configuration expérimentale pour étudier l'influence de l'angle de coupe (a) et de l'angle d'hélice (b).

sont à denture droite, le repère (tangentiel, radial, axial) correspond au repère fixe $(\vec{x}, \vec{y}, \vec{z})$ lié au dynamomètre. Les composantes tangentielle $\left(F_{x}\right)$, radiale $\left(F_{z}\right)$ et axiale $\left(F_{y}\right)$ de l'effort de coupe (dans le référentiel lié à l'outil) sont mesurées pendant l'usinage (pendant au moins dix tours). Afin de créer de l'obliquité et « d'introduire » un angle d'hélice sur une fraise à denture droite (cas de la Fig. 2b), l'outil a été déplacé radialement par rapport au tube. Par suite, un angle d'obliquité apparaît entre le vecteur vitesse $\vec{V}_{\text {c }}$ et l'arête de coupe.

\section{Résultats expérimentaux}

Toutes les données expérimentales ont été traitées grâce à un logiciel d'acquisition. Étant donné que les configurations d'essais étaient des configurations de tournage, les résultats présentés sont des valeurs moyennes des signaux.

\subsection{Influence de l'angle de coupe}

La figure 3 montre l'influence de l'angle de coupe et de l'épaisseur de coupe sur les composantes $F_{\mathrm{n}}$ et $F_{\mathrm{g}}$ de l'effort de coupe s'appliquant sur l'outil. Sur les fraises à denture droite, les composantes de l'effort de coupe dans le repère lié à la face de coupe sont obtenues à partir des composantes $F_{x}, F_{y}, F_{z}$ mesurées :

$$
\begin{aligned}
& F_{\mathrm{g}}=\cos (\gamma) \cdot F_{x}-\sin (\gamma) \cdot F_{y} \\
& F_{\mathrm{n}}=\cos (\gamma) \cdot F_{y}+\sin (\gamma) \cdot F_{x} \\
& F_{\mathrm{a}}=F_{\mathrm{z}}
\end{aligned}
$$

De plus, la composante axiale est nulle et $F_{\mathrm{g}}=F_{\mathrm{f}}$.

Premièrement, on peut noter que lorsque l'angle de coupe augmente, les composantes de l'effort diminuent. Une autre représentation de l'influence de l'angle de coupe et de l'épaisseur de coupe, ainsi que le modèle associé est donnée sur la figure 4. Ces figures montrent une évolution linéaire des efforts en fonction de l'angle de coupe et de l'épaisseur de coupe. Voici les lois de coupe du modèle de la figure 4 :

$$
\begin{aligned}
F_{n} & =\left(K_{\mathrm{n} 0}+K_{\mathrm{n} 1} \cdot\left(h-h_{0}\right)\right) \cdot b \\
& =(71+2298 \cdot(h-0,01)) \cdot b \\
F_{\mathrm{g}} & =F_{\mathrm{f}}=\left(K_{\mathrm{f} 0}+K_{\mathrm{f}} \cdot\left(h-h_{0}\right)\right) \cdot b \cdot\left(1+K_{\mathrm{n} \gamma} \cdot \gamma\right) \\
& =(79,5+2516(h-0,01)) \cdot b \cdot(1-0,029 \gamma)
\end{aligned}
$$

Les essais ont montré que la dispersion est plus importante pour les efforts de frottement que pour l'effort 

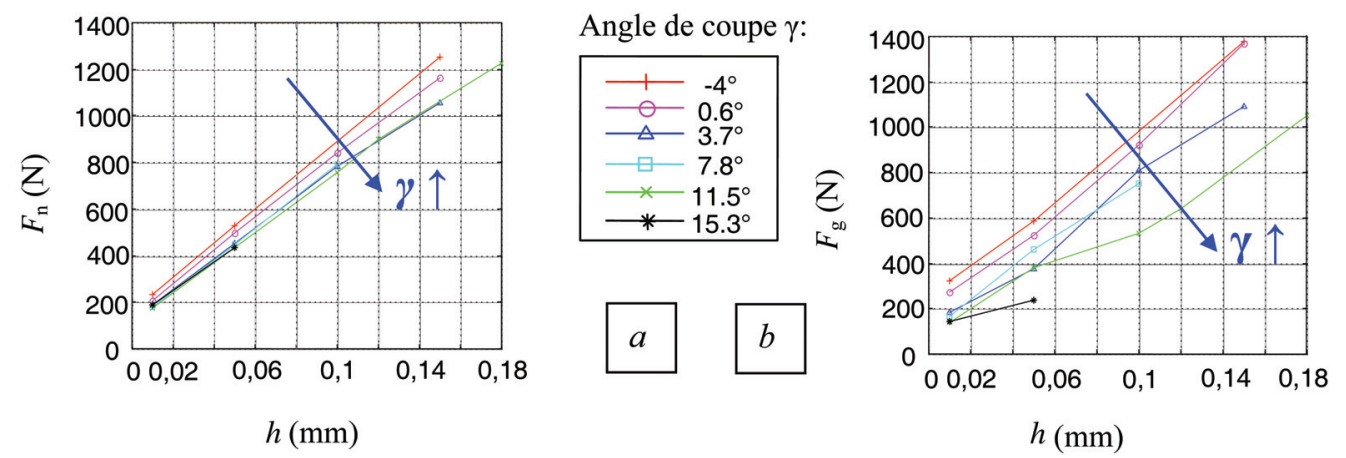

Fig. 3. Influence de l'épaisseur de coupe et de l'angle de coupe sur les composantes $F_{\mathrm{n}}(\mathrm{a})$ et $F_{\mathrm{g}}(b)$.

$a$

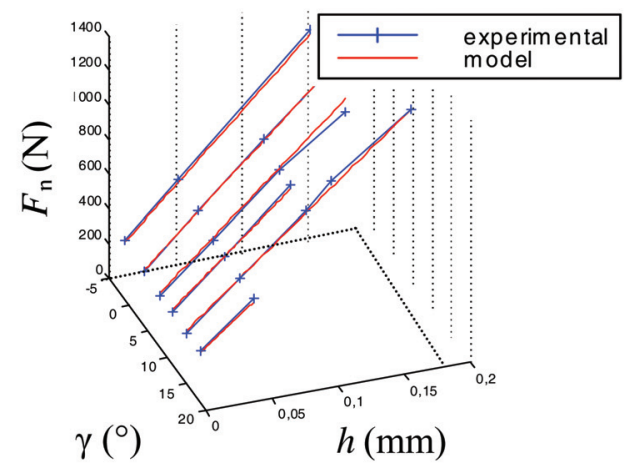

$b$

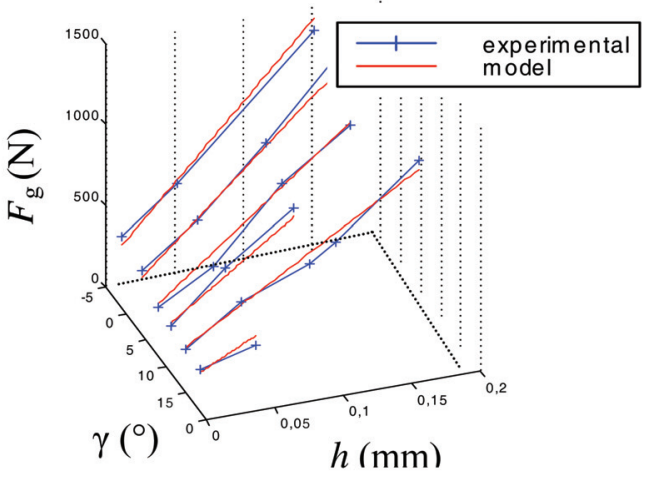

Fig. 4. Influence de l'épaisseur de coupe et de l'angle de coupe sur les composantes normale (a) et de frottement (b) et le modèle associé.

normal, ce qui pourrait être dû au fait que l'effort $F_{\mathrm{f}}$ est beaucoup plus sensible à la dégradation d'arête qui se produit pour de fortes avances que $F_{\mathrm{n}}$ (Fig. 4). Cela semble cohérent dans le contexte de l'usinage de matériaux durs, principalement en finition.

\subsection{Influence de l'angle d'hélice}

La figure 5 représente l'influence de l'angle d'hélice et de l'angle de coupe sur les composantes de l'effort appliqué sur l'outil pendant l'opération de coupe. Elles sont exprimées dans le repère lié à la face de coupe, qui est plus adéquat pour la détermination de la forme de la loi de coupe recherchée. Il semble qu'il y ait une plage optimale de variation de l'angle d'hélice.

À partir de l'évolution des efforts lors de ces deux séries d'essais préliminaires, la forme de la loi de coupe qui sera ensuite appliquée en fraisage peut être déterminée. Le modèle qui a été établi est présenté ci-après. Les variables utilisées sont : l'épaisseur de coupe $h$, la largeur de coupe $b$ et l'obliquité $\lambda_{\mathrm{s}}$. L'angle de coupe $\gamma$ est utilisé implicitement dans la détermination de l'angle $\lambda_{\mathrm{n}}$. Le modèle possède huit coefficients : $K_{\mathrm{n} \lambda_{\mathrm{s}}}, K_{\mathrm{n}_{0}}, K_{\mathrm{n}}, K_{\mathrm{n} \lambda \mathrm{s}}$, $K_{\mathrm{cf}}, K_{\mathrm{cf}_{0}}, K_{\mathrm{cf} \lambda_{\mathrm{s}}}, F_{\mathrm{f}_{0}}$.

- Calcul de l'angle d'écoulement du copeau : $\lambda_{\mathrm{e}}=K_{\lambda_{\mathrm{s}}} \cdot \lambda_{\mathrm{s}}$
- Calcul de la composante normale : $F_{\mathrm{n}}$ : $F_{\mathrm{n}}=-\left(K_{\mathrm{n}_{0}}+K_{\mathrm{n} .}\left(1+\left|\lambda_{\mathrm{s}}\right|^{K_{\mathrm{n}} \lambda_{\mathrm{s}}}\right) \cdot h\right) \cdot b$

- Évaluation du coefficient de frottement : $C_{\mathrm{f}}=\left(K_{\mathrm{cf}_{0}}+K_{\mathrm{cf}} \cdot h\right) \cdot\left(1+K_{\mathrm{cf}} \lambda_{\mathrm{s}} \cdot \lambda_{\mathrm{s}}\right)$

- Calcul de la composante $F_{\mathrm{f}}$ à partir de $C_{\mathrm{f}}$ et de la partie linéaire de $F_{\mathrm{n}}$ :

$F_{\mathrm{f}}=\left(K_{\mathrm{n}} \cdot\left(1+\left|\lambda_{\mathrm{s}}\right|^{K_{\mathrm{n} \lambda_{\mathrm{s}}}}\right) \cdot h \cdot C_{\mathrm{f}}+K_{\mathrm{f}_{\mathrm{o}}}\right) \cdot b$

- Projections sur les directions $\vec{g}$ et $\vec{a}$ :

$$
\begin{aligned}
& \lambda_{\mathrm{n}}=\arctan (\sin (\gamma)) \cdot \tan \lambda_{\mathrm{s}} \\
& F_{\mathrm{g}}=F_{\mathrm{f}} \cdot \cos \left(\lambda_{\mathrm{n}}+\lambda_{\mathrm{e}}\right) \\
& F_{\mathrm{a}}=F_{\mathrm{f}} \cdot \sin \left(\lambda_{\mathrm{n}}+\lambda_{\mathrm{e}}\right)
\end{aligned}
$$

La non-linéarité de l'évolution de la composante $F_{\mathrm{n}}$ en fonction de l'angle d'hélice $\lambda_{\mathrm{s}}$ est prise en compte par le coefficient $K_{\mathrm{n} \lambda_{\mathrm{s}}}$. Le produit h.C $C_{\mathrm{f}}$ introduit l'épaisseur de coupe au $2^{\text {nd }}$ degré dans l'expression de $F_{\mathrm{f}}$, par l'intermédiaire du coefficient : $K_{\mathrm{cf}} \cdot\left(1+K_{\mathrm{cf} \lambda_{\mathrm{s}}} \cdot \lambda_{\mathrm{s}}\right) \cdot K_{\mathrm{n}} \cdot\left(1+\left|\lambda_{\mathrm{s}}\right|^{K_{\mathrm{n} \lambda_{\mathrm{s}}}}\right)$. Le signe négatif de $K_{\text {cf }}$ rend compte de la courbure vers le bas de la courbe de $F_{\mathrm{f}}$.

Ainsi, les expressions des composantes ont été déterminées. La forme de la loi de coupe a pu être mise en place. 


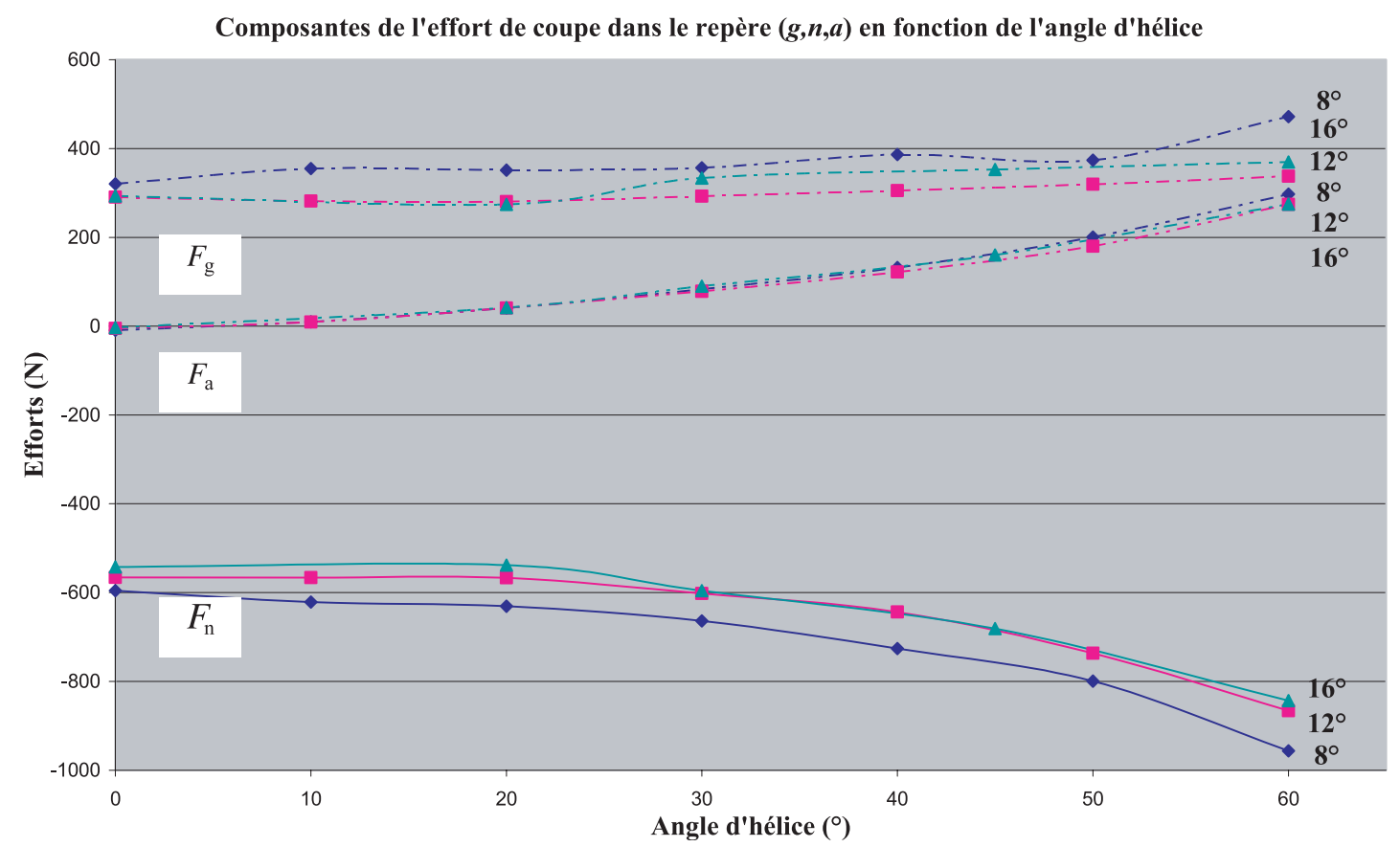

Fig. 5. Influence de l'angle d'hélice sur les composantes d'effort de coupe dans le repère $(\vec{g}, \vec{n}, \vec{a})$, pour plusieurs angles de coupe.
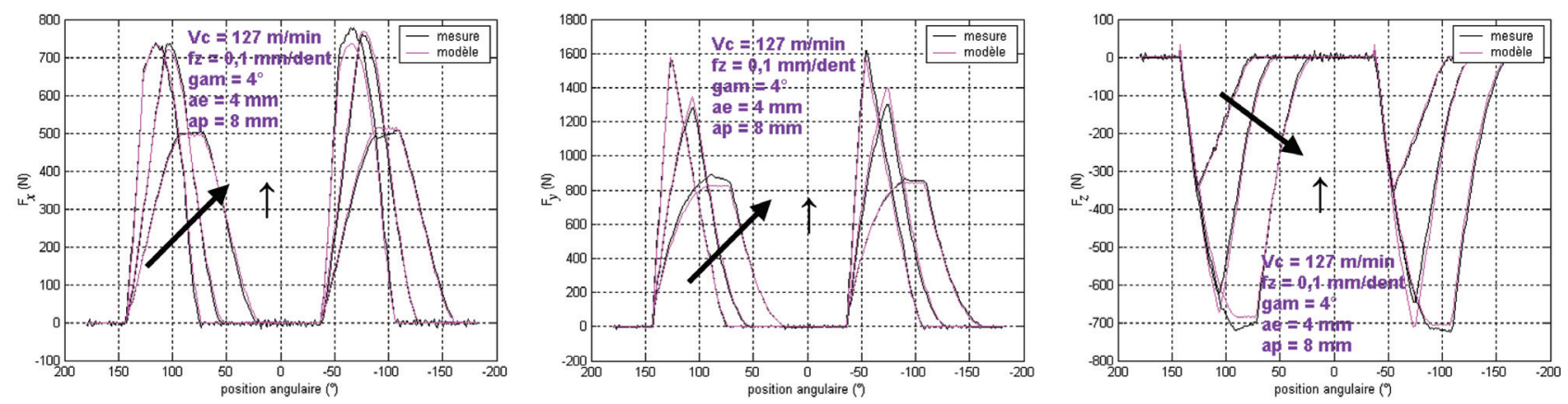

Fig. 6. Comparaison entre les efforts mesurés et les efforts modélisés dans le repère $(\vec{x}, \vec{y}, \vec{z})$.

\section{Application aux essais de fraisage}

L'étape suivante consiste en la vérification de l'application de la loi de coupe ainsi déterminée dans des conditions réelles de fraisage. Des essais ont été menés en fraisage mixte et en rainurage dans de l'acier X $38 \mathrm{CrMoV} 5$ (AISI H11) durci à $43 \mathrm{HRc}$ avec différentes fraises, sur le centre d'usinage DMG DMC $65 \mathrm{~V}$. Pendant l'usinage, les efforts ont été mesurés dans le repère de la pièce lié au dynamomètre, puis ces mesures ont permis d'évaluer les 8 coefficients du modèle établi grâce aux précédents essais. Environ 150 essais ont été réalisés avec différents angles de coupe, angles d'hélice, profondeurs de passe axiale et radiale, en opposition et en avalant. Plusieurs combinaisons des conditions de coupe listées ci-dessous ont été mises en œuvre.

- Angles de coupe $\gamma: 4^{\circ}-10^{\circ}$;

- Angles d'hélice $\lambda_{\mathrm{s}}: 0^{\circ}-20^{\circ}-40^{\circ}-60^{\circ}$;

- Avance $f=0,1 \mathrm{~mm}$.dent ${ }^{-1}$;

- Vitesse de rotation $N=2020$ tr. $\mathrm{min}^{-1}$.
- Profondeur de passe axiale $a_{\mathrm{p}}$ : 0,5-1-2-4-6-8 mm avec un engagement radial $a_{\mathrm{e}}=4 \mathrm{~mm}$;

- Profondeur de passe axiale $a_{\mathrm{p}}: 0,5-1-2-3 \mathrm{~mm}$ en rainurage $\left(a_{\mathrm{e}}=20 \mathrm{~mm}\right)$.

La figure 6 donne un exemple de résultats pour $a_{\mathrm{p}}=8 \mathrm{~mm}, a_{\mathrm{e}}=4 \mathrm{~mm}, f=0,1 \mathrm{~mm} \cdot \operatorname{dent}^{-1}, \gamma=4^{\circ}$ et $\lambda_{\mathrm{s}}=20^{\circ}-40^{\circ}-60^{\circ}$. Les essais ont été conduits avec 3 outils différents. La bonne adéquation entre le modèle et les efforts mesurés (Fig. 6) montre que le modèle est compatible avec 3 fraises différentes, d'angles d'hélice différents. Ces essais ont permis de déterminer les coefficients du modèle. Dans ce cas, l'effort de coupe peut être exprimé comme suit :

$$
\begin{aligned}
& \lambda e=0,51 \cdot \lambda_{\mathrm{s}} \\
& F_{\mathrm{n}}=-\left(18+1920 \cdot\left(1+\left|\lambda_{\mathrm{s}}\right|^{1,38}\right) \cdot h\right) \cdot b \\
& C_{\mathrm{f}}=(0,55-2,64 \cdot h) \cdot\left(1+0,0059 \cdot \lambda_{\mathrm{s}}\right) \\
& F_{\mathrm{f}}=\left(1920 \cdot\left(1+\left|\lambda_{\mathrm{s}}\right|^{1,38}\right) \cdot h \cdot C_{\mathrm{f}}+22,48\right) \cdot b
\end{aligned}
$$


En complément, dans le cas des essais de fraisage, un terme linéaire en fonction de l'épaisseur de coupe a été ajouté afin de tenir compte de l'action des arêtes frontales de la fraise et non seulement des arêtes hélicoïdales périphériques.

Les résultats présentés sont ceux correspondants aux conditions décrites précédemment. Toutefois, la même corrélation entre le modèle et les mesures a $\mathrm{pu}$ être constatée dans d'autres conditions, y compris en rainurage.

Plusieurs tendances ont également pu être dégagées suite à ces essais :

- Si l'avance par dent $f_{z}$ augmente, toutes les composantes de l'effort augmentent, ce qui s'explique par l'accroissement de la section coupée $(b \times h)$;

- Si la profondeur de passe $a_{\mathrm{p}}$ augmente, toutes les composantes de l'effort augmentent, ce qui est logique puisque plus d'éléments issus de la segmentation de la fraise sont mis en jeu dans le calcul des efforts. Cette augmentation est d'ailleurs proportionnelle à l'accroissement de la profondeur de passe axiale $a_{\mathrm{p}}$;

- Ces essais ont également permis de confirmer que, dans la zone de fonctionnement choisie, la vitesse de coupe $V_{\mathrm{c}}$ n'a pas d'influence sur les efforts; d'où sa non-prise en compte dans la loi de coupe;

- Le mode de fraisage ne semble pas avoir d'influence sur la composante axiale des efforts. Par contre, les deux autres composantes $\left(F_{x}=\right.$ suivant la direction d'avance et $F_{y}=$ orthogonale à la direction d'avance et à l'axe de l'outil) varient en sens opposé suivant le mode de fraisage adopté;

- Enfin, si l'angle d'hélice s'accroît, la composante axiale $F_{z}$ augmente mais $F_{x}$ et $F_{y}$ diminuent. Ceci provient vraisemblablement du changement d'orientation de l'effort résultant dans l'espace et par conséquent des projections sur les trois directions du repère.

\section{Conclusions et perspectives}

Les résultats présentés dans cet article montrent que la forme du modèle déterminée grâce à la première série d'essais s'applique au processus de fraisage. D'autres formes de relation de coupe pourraient être envisagées, en augmentant le degré des équations ou leur non-linéarité par exemple. Mais ceci entraînerait une augmentation du nombre de coefficients à déterminer. D'où des essais plus nombreux d'une part, et le risque d'introduire de l'instabilité dans le modèle d'autre part. Notre objectif serait donc plus d'améliorer encore ce modèle en donnant par exemple, des ordres de priorité dans la détermination des coefficients afin de limiter les calculs en fonction de la précision demandée : il n'est pas nécessaire de « dépenser » du temps de calcul pour déterminer des coefficients de faible influence dans le modèle si la valeur finale d'effort recherchée n'est pas très précise.

La détermination de l'évolution temporelle des efforts pendant des opérations d'usinage grâce à un nombre d'essais réduit, pour un couple outil-matière donné, et l'évaluation de certains coefficients qui apparaissent dans l'étude de la stabilité de la coupe peuvent donc être envisagées. Ce modèle pourrait également être intéressant en conception d'outil pour créer une géométrie adaptée à une opération d'usinage donnée avec, par exemple, des paramètres géométriques variables le long de l'arête de coupe (angles de coupe et d'hélice). L'étude présentée ici permet la détermination des efforts de coupe pendant une opération d'usinage en fonction non seulement des conditions de coupe mais aussi de la géométrie d'outil. Pour le moment, seuls l'angle de coupe et l'angle d'hélice ont été étudiés, pour un matériau usiné et un outil donné. Il serait intéressant de réaliser d'autres essais afin d'introduire de nouveaux paramètres dans la loi de coupe, tels que le diamètre, le nombre de dents, ou encore la préparation d'arête. De plus, d'autres géométries d'outils devront être envisagées, telles que des fraises hémisphériques ou rayonnées.

Remerciements. Les auteurs tiennent à remercier la société Prototyp S.A. pour sa participation à cette étude. La participation du CEA Valduc est également grandement remerciée.

\section{Références}

[1] E.J.A. Armarego, E. Brown, The Machining of Metals, Prentice Hall Inc., 1969

[2] C. Rubenstein, The Mechanics of Continuous Chip Formation in Oblique Cutting in the Absence of Chip distortion, Part 1 : Theory, Int. J. Machine Tools and Manufacture 23 (1983) 11-20

[3] J.A. Bailey, G. Boothroyd, Critical Review of Some Previous Work on the Mechanics of the Metal-Cutting Process, Transactions of ASME, 67, 1968

[4] Y. Altintas, P. Lee, A general mechanics and dynamics model for helical end mills, Annals of the CIRP 45/1 (1996) 59-64

[5] C.E. Becze, M.A. Elbestawi, A chip formation based analytic force model for oblique cutting, Int. J. Machine Tools and Manufacture 42 (2002) 529-538

[6] E.J.A. Armarego, N.P. Deshpande, Force prediction models and CAD/CAM software for helical tooth milling processes. I. Basic approach and cutting analyses, Int. J. Prod. Res. 31(8) (1993) 1991-2009

[7] E.J.A. Armarego, N.P. Deshpande, Force prediction models and CAD/CAM software for helical tooth milling processes. II. Peripheral milling operations, Int. J. Prod. Res. 31 (1993) 2319-2336

[8] E.J.A. Armarego, N.P. Deshpande, Force prediction models and CAD/CAM software for helical tooth milling processes. III. End-milling and slotting operations, Int. J. Prod. Res. 32 (1994) 1715-1738

[9] E.J.A. Armarego, R.C. Whitfield, Computer Based Modelling of Popular Machining Operations for Force and Power Predictions, Annals of CIRP 34/1 (1985) 65-69

[10] S. Jayaram, S.G. Kapoor, R.E. De Vor, Estimation of the specific cutting pressures for mechanistic cutting force models, Int. J. Machine Tools and Manufacture 41 (2001) $265-281$ 
[11] J.J. Junz Wang, C.M. Zheng, An analytical force model with shearing and ploughing mechanisms for end milling, Int. J. Machine Tools and Manufacture 42 (2002) 761-771

[12] P.L.B. Oxley, Mechanics of Machining, Ellie Horwood Limited, Chichester, 1989

[13] H.Z. Li, W.B. Zhang, X.P. Li, Modelling of cutting forces in helical end milling using a predictive machining theory, Int. J. Mec. Sci. 43 (2001) 1711-1730

[14] Y. Altintas, S. Engin, Generalized modeling of mechanics and dynamics of milling cutters, Annals of the CIRP 50/1 (2001) 25-30

[15] F. Lapujoulade, K. Raïssi, T. Mabrouki, Modélisation et simulation temporelle du comportement en fraisage des plaques minces en fraisage latéral de finition, Proceedings des $2^{\text {es }}$ Assises Machines et Usinage à Grande Vitesse, Lille, France, 2002, pp. 251-259

[16] J.H. Ko, W.S. Yun, D.W. Cho, K.F Ehmann, Development of a virtual machining system, Part 1 : Approximation of the size effect for cutting force prediction, Int. J. Machine Tools and Manufacture, 42 (2002) 1595-1605

[17] French standard AFNOR XP E 66-520-5, Domaine de fonctionnement des outils coupants. Couple outil-matière, ISSN 0335-3931, 1995 